\title{
A description of the basic system of components in pregnant mice sera responsible for 'early pregnancy factor' activity
}

\author{
C. Orozco, I. Q. Clark, I. E. Cock and F. M. Clarke* \\ Faculty of Science and Technology, Griffith University, Nathan, Brisbane 4111, Australia
}

\begin{abstract}
The supernatant and pellet retentate fractions obtained from sera from pregnant mice by ammonium sulfate fractionation and extensive dialysis cannot alone induce increased rosette inhibition titres in the rosette inhibition assay. In combination, however, the mixtures can induce increased rosette inhibition titres mimicking the activity of pregnancy sera previously ascribed to the early pregnancy factor. The studies described herein demonstrated that the supernatant retentate fractions derived from sera of pregnant mice were functionally equivalent to a platelet-activating factor (PAF) or a serum stimulus because they stimulated the spleen cells used in the assay to produce active moieties and cooperated with pure thioredoxin in allowing for expression of activity. Conversely, the pellet retentate fractions obtained from sera of pregnant mice were shown to be functionally equivalent to thioredoxin in that they cooperated with a PAF stimulus to allow for the expression of increased rosette inhibition titres. The supernatant retentate fractions obtained from sera from male mice were found to be functionally equivalent to the corresponding supernatant retentate fractions obtained from sera from pregnant mice in stimulating the production of active moieties and in cooperating with thioredoxin or the pellet fractions derived from sera from pregnant mice in allowing for increased rosette inhibition titres. The pellet retentate fractions obtained from male mouse sera, however, were not functionally equivalent to either thioredoxin or the corresponding pellet retentate fractions obtained from pregnancy sera. Consideration of these data led to a basic description of the system of components in pregnancy sera which is responsible for the expression of early pregnancy factor activity.
\end{abstract}

\section{Introduction}

The susceptibility of early pregnancy factor activity in sera of pregnant animals to ammonium sulfate fractionation is well known (Clarke et al., 1980; Sueoka et al., 1989). Clark et al. (1994) reinvestigated the effects of ammonium sulfate fractionation. Treatment of sera from pregnant mice with $40 \%$ ammonium sulfate was shown to liberate low molecular mass active moieties from their association with macromolecular serum components. After centrifugation, these active moieties partition into the supernatant fraction, while the macromolecular components to which they are bound partition into the pellet fraction. The macromolecular components of the supernatant and pellet fractions freed of any association with these low molecular mass moieties by extensive dialysis, cannot alone induce increased rosette inhibition titres. In combination, however, components in the dialysed pellet retentate fraction cooperate with components in the dialysed supernatant retentate fraction to allow the expression of increased rosette inhibition titres when applied to fresh spleen cells in the rosette inhibition assay. In two-step incubation protocols, a prescribed order of addition must be followed if this cooperative effect is

\footnotetext{
*Correspondence
}

Received 24 May 1993. to be observed, namely supernatant retentate fraction applied first, followed by pellet retentate fraction in the second step, but not vice versa (Clark et al., 1994).

The features of this cooperation are similar to findings (Clarke et al., 1991) that combinations of normal serum plus thioredoxin or platelet-activating factor (PAF) plus thioredoxin can also induce increased rosette inhibition titres. None of these components is effective alone. Again, the effects are observed in two-step protocols only if prescribed orders of addition are followed, in these cases serum or PAF are applied in the first step followed by thioredoxin. Serum or PAF act on the spleen cell population to stimulate the release of active moieties (termed S2 factors) but at the same time to render potential responding cell populations refractory to the action of these moieties and so no increased rosette inhibition titres are observed. Thioredoxin, if present, or if subsequently added, prevents or reverses this refractory state allowing the expression of increased rosette inhibition titres where none is observed in its absence. These observations suggest that the supernatant and pellet retentate in combination may act in an analogous manner, that is with components in the supernatant retentate fraction stimulating the production of potentially active moieties but at the same time inducing a refractory state, whereas components in the pellet retentate fraction act in a permissive manner, analogous to the reversal of the refractory 
state by thioredoxin, ensuring the expression of increased rosette inhibition titres.

If this is the case, there are a number of propositions that can be tested experimentally. It would be predicted that (i) components in the extensively dialysed supernatant retentate fraction should cooperate with pure thioredoxin to allow for increased rosette inhibition titres; (ii) conversely, components in the extensively dialysed pellet retentate fraction from sera of pregnant mice should cooperate with a PAF stimulus to allow for increased rosette inhibition titres and (iii) components in the extensively dialysed supernatant fraction should stimulate S2 factor production. Experiments to test these and other related propositions all concerned with giving further definition to the early pregnancy factor system of components present in pregnancy sera are described.

\section{Materials and Methods}

\section{Collection of sera and ammonium sulfate fractionation}

Sera were prepared from the blood of male mice and female mice (at days 2 and 10 of pregnancy) as described by Clark et al. (1994). Individual serum samples were fractionated with $40 \%$ ammonium sulfate and the resulting supernatant and pellet fractions were extensively dialysed against PBS using nominal $8 \mathrm{kDa}$ cut-off dialysis membranes. The dialysis procedures were as described for Expt 1 in Clark et al., 1994, that is, each individual sample was dialysed alone in a separate container against three successive changes of 100-fold excess volumes of PBS. After the third dialysis step, the retentates were collected and stored in suitable aliquots at $-30^{\circ} \mathrm{C}$. The extensively dialysed supernatant and pellet retentate fractions obtained from pregnancy sera (day 2 or day 10) were designated as $\mathrm{pSr}$ and $\mathrm{pPr}$, respectively, while those from male mouse sera were designated as $\mathrm{mSr}$ and $\mathrm{mPr}$, respectively.

\section{Reagents}

Synthetic platelet-activating factor (1-O-alkyl-2-acetylsn-glycero-3-phosphocholine, C18) was obtained from Boehringer, Mannheim, Germany. Stock and working solutions were prepared as described previously (Orozco et al., 1990). Human recombinant thioredoxin was produced, purified and stored as described by Clarke et al. (1991).

\section{Spleen cell preparation, cell incubations and the rosette inhibition assay}

These preparations and procedures were performed as described by Clark et al. (1994).

\section{Production of active $S 2$ fractions}

Mouse spleen cells $\left(15 \times 10^{6}\right)$ were incubated in the presence of test samples $(0.5 \mu \mathrm{l})$ in $200 \mu \mathrm{l}$ of PBS. After incubation for $30 \mathrm{~min}$ at $37^{\circ} \mathrm{C}$ the cells were sedimented, washed twice in Hank's balanced salt solution, resuspended in $200 \mu \mathrm{l}$ PBS and incubated for a further $30 \mathrm{~min}$ at $37^{\circ} \mathrm{C}$. The cells were sedimented and the supernatants, designated the S2 fractions (Orozco et al., 1990), were collected and immediately taken for testing in the rosette inhibition assay using fresh mouse spleen cells.

\section{Results}

Samples of sera from day 2 and day 10 pregnant mice as well as sera from male mice were fractionated with $40 \%$ ammonium sulfate and the resulting pellet and supernatant fractions were extensively dialysed in separate containers to yield pellet and supernatant retentate fractions. As shown by Clark et al. (1994), these individual retentate fractions were unable to induce increased rosette inhibition titres when tested over a wide dose range. However, when the fractions derived from sera of pregnant mice were combined, the mixtures were very effective in inducing increased rosette inhibition titres. These preparations were used in the following experiments to investigate their modes of action in cooperating in the rosette inhibition assay.

Cooperation between the pellet retentate fractions derived from sera of pregnant mice and a PAF stimulus

The observation (Clark et al., 1994) that the pellet retentate fractions derived from sera of pregnant mice could act subsequently to the corresponding supernatant retentate fractions in a two-step incubation protocol (but not vice versa) suggested that the pellet retentates derived from sera of pregnant mice may be acting in a permissive manner analogous to that previously demonstrated for thioredoxin (Clarke et al., 1991). It would thus be predicted that these fractions should also cooperate with a PAF stimulus, as does thioredoxin. The abilities of the pellet (and the supernatant) retentate fractions to cooperate with a PAF stimulus in inducing increased rosette inhibition titres were therefore investigated by incubating spleen cells with combinations of $5 \mathrm{nmol} \mathrm{PAF} \mathrm{l}^{-1}$ and $2 \mu \mathrm{l}$ of 1:10 serial dilutions of the individual samples before testing in the rosette inhibition assay. The pellet retentate fractions from sera from both day 2 and day 10 pregnant mice (Fig. Ib), but not from sera of male mice (Fig. Id), mimicked the action of thioredoxin (Clarke et al., 1991) in cooperating with PAF to allow for the expression of increased rosette inhibition titres where none was observed in their absence. Combinations of PAF with the supernatant retentate fractions from either the sera of pregnant mice (Fig. 1a) or of male mice (Fig. Ic) do not induce increased rosette inhibition titres. The dose-response curves of PAF in cooperating with a fixed amount of the pellet retentate fraction derived from sera from pregnant mice or with a fixed concentration $\left(1 \mathrm{nmol} 1^{-1}\right.$ ) of pure thioredoxin to allow for increased rosette inhibition titres are given (Fig. 2). In these combinations, PAF was very effective down to very low doses. However, it should be noted that when tested alone over the same wide range of initial concentrations, PAF was not effective in inducing increased rosette inhibition titres (Fig. 2). Cavanagh et al. (1991) reported that PAF alone was effective, albeit within a very narrow and extremely low dose response range $\left(0.2 \mathrm{fmol} \mathrm{l}^{-1}-2 \mathrm{amol} \mathrm{l}^{-1}\right)$. Such an effect was not observed in the studies reported here; in repeated experiments 

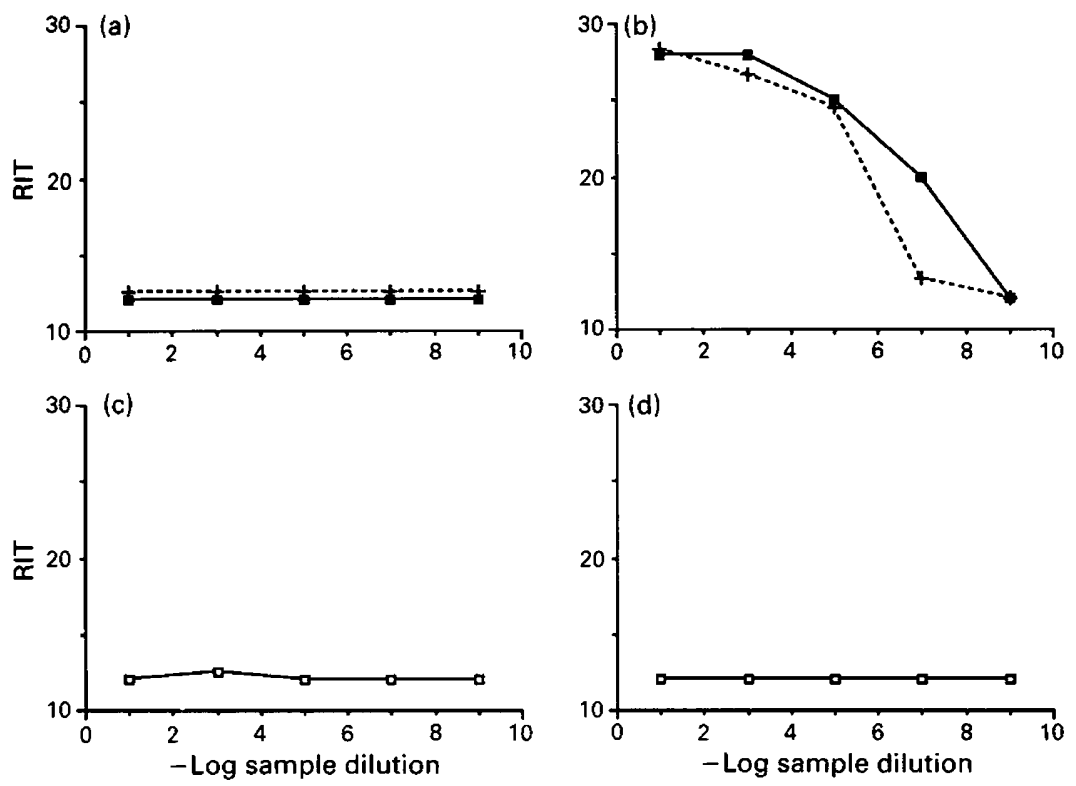

Fig. 1. Dose-response curves of supernatant and pellet retentate fractions $(2 \mu \mathrm{l}$ of indicated dilutions) in cooperating with platelet-activating factor (PAF) $\left(5 \mathrm{nmol} \mathrm{l}^{-1}\right.$ ) in the rosette inhibition assay. (a) Supernatant and (b) pellet retentate fractions derived from sera from ( $\boldsymbol{C}$ ) day 2 and $(+)$ day 10 pregnant mice; (c) supernatant and (d) pellet retentate fractions from sera from male mice. Results are means of triplicate determinations. RIT: rosette inhibition titre.

in which PAF alone was applied to the mouse spleen cell preparations over a wide range of initial concentrations, no increased rosette inhibition titres were observed (Fig. 2).

\section{Cooperation between thioredoxin and the supernatant retentate} fractions from sera from pregnant and male mice

The supernatant retentate fractions may be acting in a manner analogous to a PAF stimulus. The capacities of the supernatant retentate fractions (and also the pellet retentate fractions as controls) to cooperate with thioredoxin were tested by incubating spleen cells with $2 \mu \mathrm{l}$ of 1:10 dilutions of the individual supernatant (or pellet) retentate fractions in combination with serial dilutions of thioredoxin. The cells were subsequently tested in the rosette inhibition assay. The supernatant retentate fractions from the sera of both pregnant and male mice (Fig. 3a, c) were effective in combining with thioredoxin to allow the expression of increased rosette inhibition titres. In two-step incubation protocols, the combinations were effective only if the supernatant retentate fractions were added in the first step (data not shown). However, the combinations of thioredoxin with the pellet retentate fractions were totally without effect and only control rosette inhibition titre values were observed (Fig. 3b, d).

Stimulation of the production of active $S 2$ fractions by the supernatant retentate fractions from sera from pregnant mice and sera from male mice

As PAF and mouse sera were shown (Orozco et al., 1990; Clarke et al., 1991) to stimulate mouse spleen cells to produce

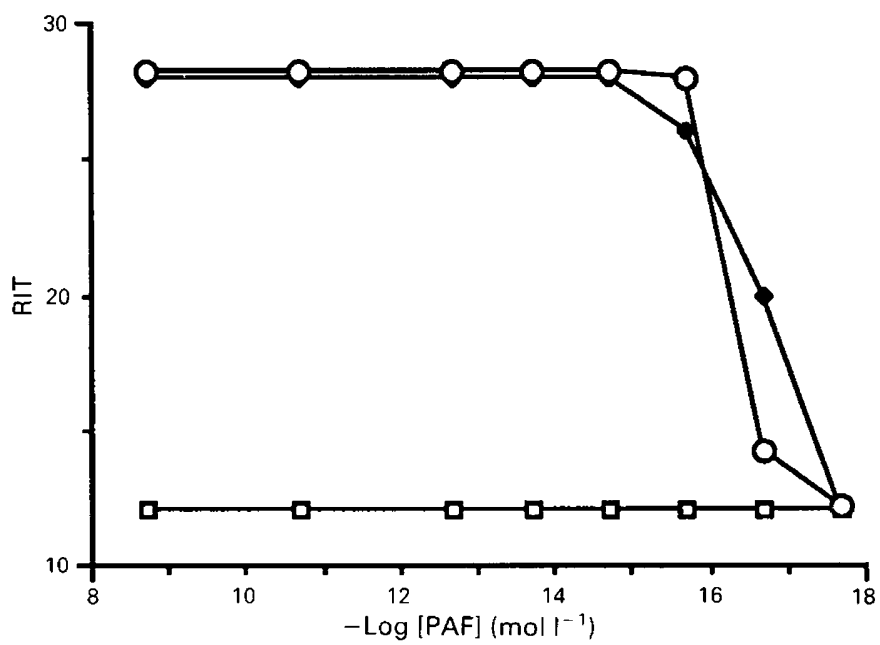

Fig. 2. Dose-response curve of platelet-activating factor (PAF) in the rosette inhibition assay $(\square)$ alone, $(O)$ in combination with $1.0 \mathrm{nmol}$ thioredoxin $\mathrm{l}^{-1}$ or $(\bullet)$ in combination with $0.2 \mu$ l pellet retentate fraction derived from serum of day 2 pregnant mice. Results are means of triplicate determinations.

active S2 fractions, and as the supernatant retentate fractions were shown to mimic PAF and sera in their capacity to cooperate with thioredoxin, experiments were undertaken to examine whether these supernatant retentates also mimicked $\mathrm{PAF}$ and whole sera in stimulating the production of active S2 fractions. Mouse spleen cells were incubated with $2 \mu \mathrm{l}$ of I:10 dilutions of supernatant (and as control pellet) retentate fractions for $30 \mathrm{~min}$ at $37^{\circ} \mathrm{C}$. After centrifugation, the cells were washed twice and then resuspended in PBS for a second 

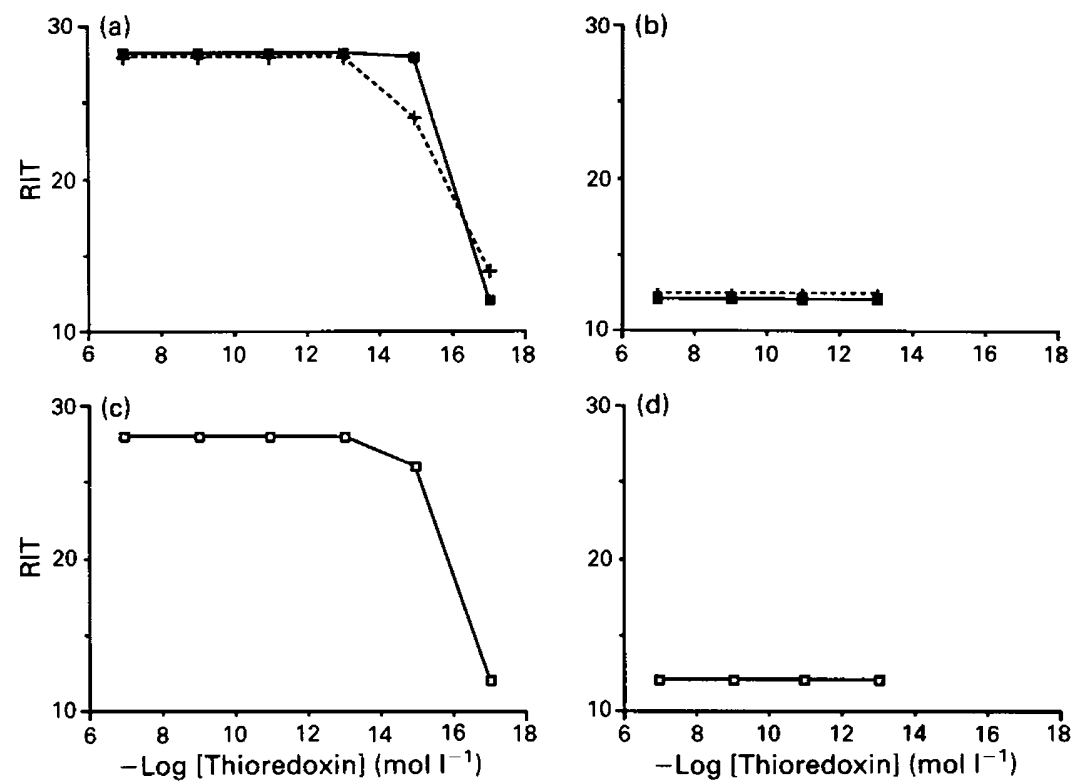

Fig. 3. Ability of supernatant and pellet retentate fractions $(0.2 \mu \mathrm{l})$ to cooperate with indicated concentrations of thioredoxin in the rosette inhibition assay. (a) Supernatant and (b) pellet retentate fractions from sera of pregnant mice from ( $\boldsymbol{D})$ day 2 and $(+)$ day 10 of pregnancy. (c) Supernatant and (d) pellet retentate fractions from sera derived from male mice. Results are means of triplicate determinations. RIT: rosette inhibition titre.

incubation at $37^{\circ} \mathrm{C}$ for $30 \mathrm{~min}$. After centrifugation the supernatant (S2) fractions were collected and serial dilutions applied to fresh spleen cells for testing in the rosette inhibition assay. The supernatant retentate fractions from sera of both pregnant and male mice stimulated the production of S2 fractions which were extremely potent in inducing increased rosette inhibition titres when applied to fresh spleen cells (Fig. 4a, c). The S2 fractions derived from cells incubated with the pellet retentate fractions of sera from either pregnant or male mice were not active in the rosette inhibition assay (Fig. $4 \mathrm{~b}, \mathrm{~d}$ ).

Cooperation between the supernatant retentate fractions from sera from male mice with the pellet retentate fractions from sera of pregnant mice

As the supernatant retentate fractions derived from sera from male mice were shown to be functionally equivalent to those from sera from pregnant mice in cooperating with thioredoxin (Fig. 3) and in stimulating the production of active S2 fractions (Fig. 4), it was of interest to determine whether they could also cooperate with the pellet retentate fractions of sera from pregnant mice in inducing increased rosette inhibition titres. Consequently, the various combinations of pellet and supernatant retentate fractions derived from sera from male and pregnant mice were tested. As shown by Clark et al. (1994) and the study reported here (Fig. 5a), the combination of the pellet and supernatant retentate fractions from sera from pregnant mice was very effective in inducing increased rosette inhibition titres. It can be seen that the supernatant retentate fraction of sera from male mice was just as effective as the corresponding supernatant retentate fraction derived from sera from pregnant mice in that it could also combine with the pellet retentate fraction of sera from pregnant mice in inducing increased rosette inhibition titres (Fig. 5b). However, the pellet retentate fraction of serum from male mice was not equivalent to the corresponding fraction derived from serum of pregnant mice, as it could not cooperate with either type of supernatant retentate fraction in inducing increased rosette inhibition titres (Fig. 5c, d). This finding contrasts with the permissive action of the pellet retentate fractions of sera from pregnant mice, which cooperate with either type of supernatant retentate fraction to allow for increased rosette inhibition titres (Fig. 5a, b).

\section{Discussion}

The results of the studies reported here allow us to present a model (Fig. 6) for the modes of action of the supernatant and pellet retentate fractions derived from sera from pregnant mice in cooperating to induce increased rosette inhibition titres, and to compare and contrast these actions with those of the corresponding fractions derived from sera from male mice. The data clearly indicate that supernatant retentate fractions derived from sera from pregnant mice act in a manner analogous to that demonstrated for PAF (Orozco et al., 1990) and normal serum (Clarke ef al., 1991), that is they stimulate the spleen cell population to produce active moieties ( $\mathrm{S} 2$ factors), but at the same time render the potential responding cell population(s) refractory to their action and thus when applied alone increased rosette inhibition titres are not observed. However, pellet retentate fractions derived from sera of pregnant mice act in a manner analogous to pure thioredoxin. Although ineffective when applied alone, if applied with or 

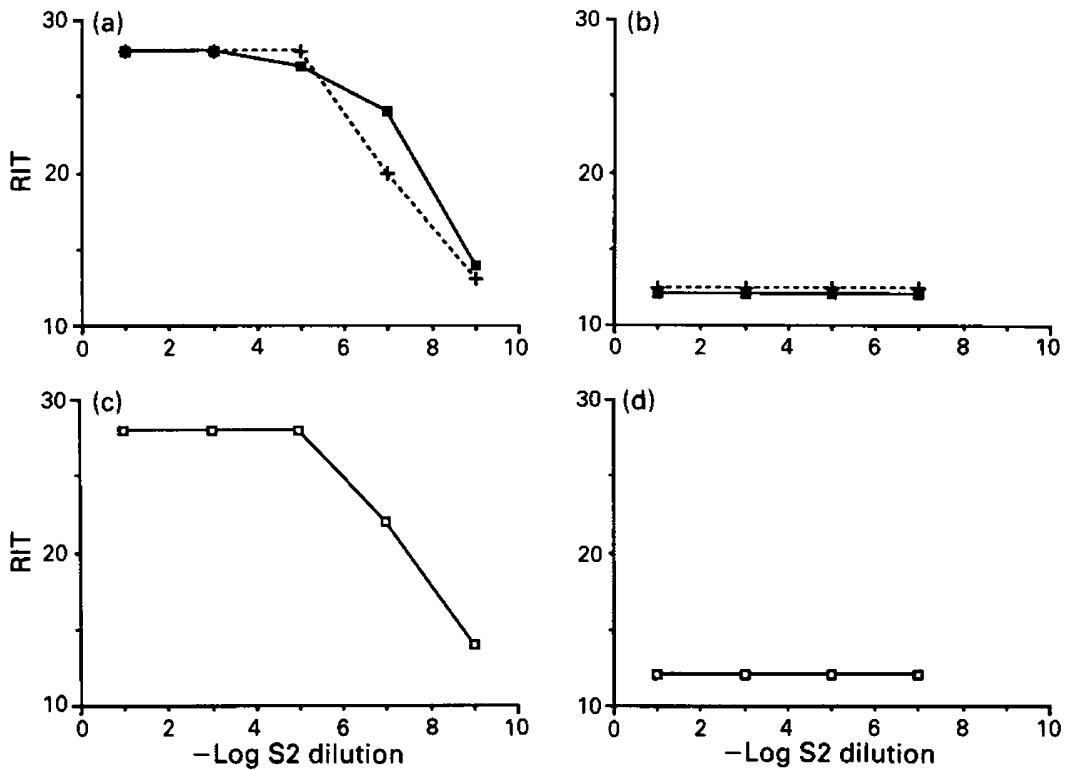

Fig. 4. Dose-response curves in the rosette inhibition assay of S2 fractions obtained after stimulation of mouse spleen cells with supernatant and pellet retentate fractions. (a) Supernatant retentate fractions and (b) pellet retentate fractions derived from sera from ( $\boldsymbol{\square}$ ) day 2 and ( + ) day 10 pregnant mice; (c) supernatant and (d) pellet retentate fractions derived from sera from male mice. Results are means of triplicate determinations. RIT: rosette inhibition titre.
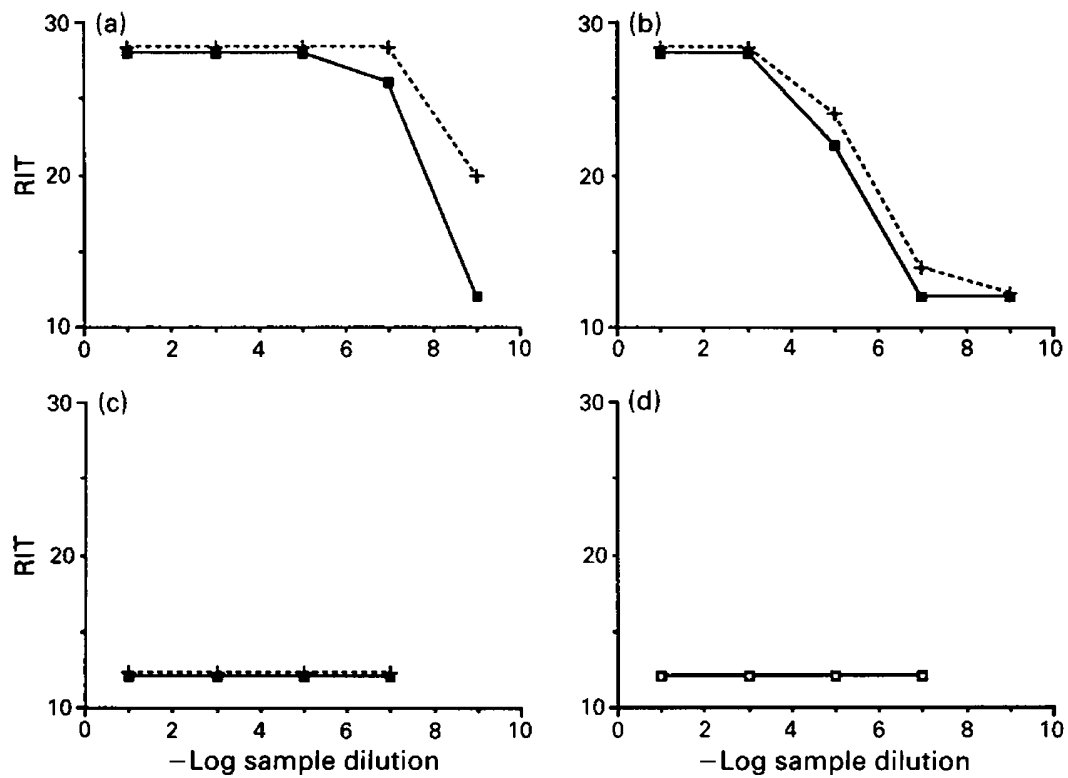

Fig. 5. Dose-response curves in the rosette inhibition assay of mixtures made by combining equal amounts of supernatant and pellet retentate fractions. (a) Supernatant retentates combined with pellet retentate fractions from sera from (D) day 2 and (+) day 10 pregnant mice; (b) supernatant retentate fraction from sera from male mice combined with pellet retentate fractions from sera derived from ( $\boldsymbol{\square})$ day 2 and (+) day 10 pregnant mice; (c) supernatant retentate fractions from sera derived from (a) day 2 or $(+)$ day 10 pregnant mice combined with pellet retentate fraction from serum from male mice; (d) supernatant retentate fraction combined with pellet retentate fraction both from serum of male mice. Results are means of triplicate determinations. 


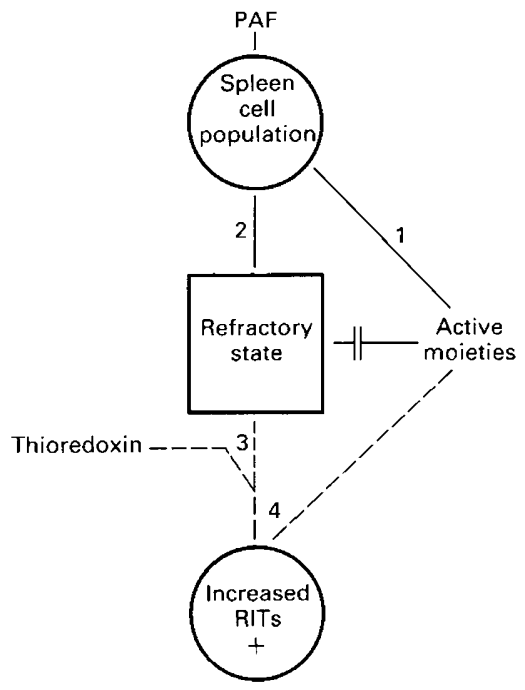

(a)

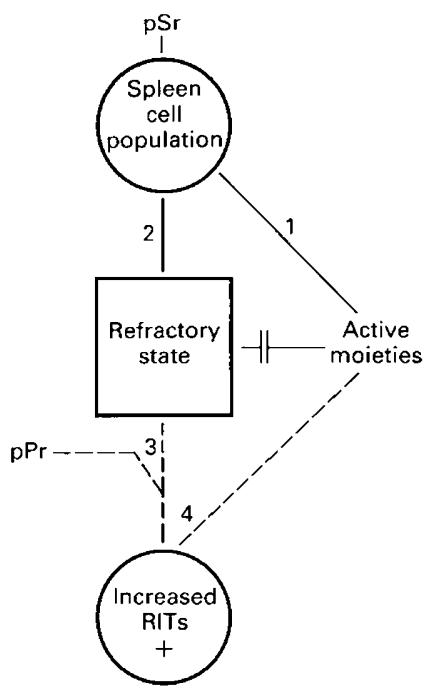

(b)

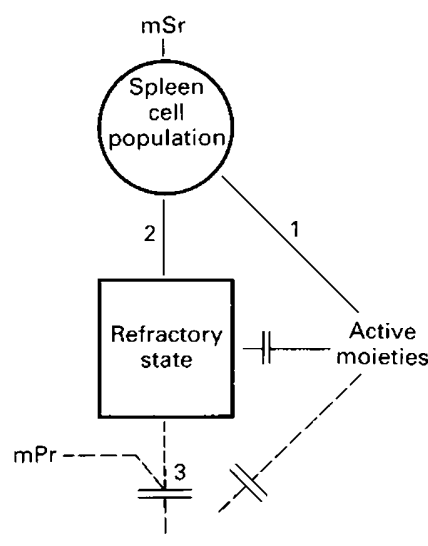

(c)

Fig. 6. (a) Model of action of platelet activating factor (PAF) and thioredoxin in cooperating to allow for the expression of increased rosette inhibition titres (RITs). (1) PAF stimulates cells to produce active moieties but (2) PAF also induces a refractory state preventing active moieties from exerting their effects. Addition of thioredoxin (3) reverses the refractory state allowing active moieties to act (4) in inducing increased rosette inhibition titres; (b) model of action of supernatant ( $\mathrm{pSr}$ ) and pellet $(\mathrm{pPr}$ ) retentate fractions derived from serum from pregnant mice in cooperating to allow for expression of increased rosette inhibition titres. The supernatant retentate fraction functions in a manner analogous to PAF (1) stimulating the production of active moieties but at the same time (2) inducing a refractory state. The pellet retentate fraction functions in a manner analogous to thioredoxin by (3) reversing the refractory state allowing (4) the active moieties to exert their effect; (c) model of action of supernatant $(\mathrm{mSr})$ and pellet $(\mathrm{mPr})$ retentate fractions derived from serum of male mice in the rosette inhibition assay. The supernatant retentate fraction acts in a manner ( 1 and 2 ) analogous to that of PAF and the corresponding fraction derived from sera of pregnant mice. The pellet retentate fraction of sera from male mice, however, is not functional (3) and does not act to reverse the refractory state and rosette inhibition titres are not increased.

subsequent to appropriate cell stimuli such as PAF or the supernatant retentate fractions, they prevent or reverse the refractory state, allowing the expression of increased rosette inhibition titres. The capacities of supernatant retentate fractions derived from sera of pregnant mice to stimulate the production of active S2 fractions and to cooperate with pure thioredoxin and the demonstrated capacity of the pellet retentate fractions derived from sera of pregnant mice to cooperate with a PAF stimulus are consistent with these conclusions.

The supernatant retentate fractions derived from sera from male mice would appear to be functionally equivalent to the corresponding fractions derived from sera from pregnant mice in that they also stimulated the production of active S2 fractions and cooperated with both thioredoxin and the pellet retentate fractions derived from sera from pregnant mice to induce increased rosette inhibition titres. The observation of these particular functional capacities in a fraction derived from sera from male mice is consistent with our previous observations (Clarke et al., 1991) that unfractionated sera from male mice also express these capacities, that is the ability to cooperate with thioredoxin and to stimulate the production of an active $S 2$ fraction. The results reported here indicate that the component(s) responsible partition into the supernatant retentate fractions following ammonium sulfate fractionation of sera from male mice.

In contrast to the functional equivalence of the supernatant retentate fractions, the pellet retentate fractions derived from sera from male mice and from pregnant mice were not equivalent. Although the pellet retentate fractions derived from sera of pregnant mice function in a permissive manner in combination with PAF or the supernatant retentate fractions, those from sera from male mice do not. This permissive capacity of the pellet retentate fractions derived from sera of pregnant mice was equivalent to that of pure thioredoxin and this finding implies that functional thioredoxin molecules may be present in these fractions. These conclusions are in agreement with our previous suggestions (Clarke et al., 1991; Clarke, 1992) that a fundamental difference between sera from pregnant and non-pregnant mice may be the presence in the former of functional forms of thioredoxin or thioredoxin-like molecules. The present results suggest that such molecules partition into the pellet fraction on ammonium sulfate fractionation of pregnancy sera and this conclusion is supported by recent adsorption studies using polyclonal and monoclonal anti-thioredoxin antibodies (F. M. Clarke and C. Orozco, unpublished).

From the studies described here and by Clark et al. (1994), the effect of ammonium sulfate on the capacity of sera from pregnant mice to induce increased rosette inhibition titres can be explained in principle in the following terms. Pregnancy sera contain stimulatory and permissive components and potentially active low molecular mass moieties, most or the most potent of which associate with the latter. Sera from non-pregnant mice lack the permissive components and possibly the low molecular mass components. On ammonium sulfate fractionation of 
pregnancy sera, the low molecular mass moieties are freed from their association with the permissive components and the low molecular mass moieties partition into the supernatant fraction with the stimulatory components. The permissive, thioredoxinlike components, partition into the pellet fraction. On dialysis the low molecular mass active moieties are removed from the supernatant fraction. The stimulatory fraction induces the production of potentially active moieties when applied to mouse spleen cells in the rosette inhibition assay but also induces a refractory state, and so alone cannot induce increased rosette inhibition titres. On addition of the pellet retentate fraction which contains functional thioredoxin, or thioredoxin-like permissive molecules, this refractory state is reversed or prevented and the combination therefore allows for the expression of increased rosette inhibition titres. There is considerable evidence to support the conclusion that functional thioredoxin or thioredoxin-like molecules are key components of the pellet retentate fractions of sera from pregnant but not from nonpregnant mice. With respect to the stimulatory components of the supernatant fractions and the low molecular mass active moieties, the studies reported here establish their existence and relevance, and further studies are now needed to establish their identity.

This work was funded in part by the National Health and Medical Research Council of Australia and by the Lalor Foundation.

\section{References}

Cavanagh AC, Rolfe BE, Athanasas-Platsis S, Quinn KA and Morton H (1991) Relationship between early pregnancy factor, mouse embryo-conditioned medium and platelet-activating factor Journal of Reproduction and Fertility 93 $355-365$

Clark IQ, Orozco C, Cock IE and Clarke FM (1994) The 'early pregnancy factor' revisited: the effect of ammonium sulfate on the capacity of pregnant mouse sera to express activity in the rosette inhibition assay Joumal of Reproduction and Fertility $100279-289$

Clarke FM (1992) Identification of molecules and mechanisms involved in the 'Early Pregnancy Factor' system Reproduction, Fertility and Development 4 423-433

Clarke FM, Morton H, Rolfe BE and Clunie GJA (1980) Partial characterisation of early pregnancy factor in sheep Journal of Reproductive Immunology 2 151-162

Clarke FM, Orozco C, Perkins AV, Cock IE, Tonissen KF, Robins AJ and Wells JRE (1991) Identification of molecules involved in the 'early pregnancy factor' phenomenon Journal of Reproduction and Fertility 93 525-539

Orozco C, Cock I, Perkins AV and Clarke FM (1990) Platelet activating factor and serum components from oestrous mice co-operate to mimic the activity of 'early pregnancy factor' in the rosette inhibition assay Journal of Reproduction and Fertility 88 447-457

Sueoka K, Dharmarajan AM, Miyazaki T, Atlas SJ and Wallach EE (1989) In vivo and in vitro determination of components of rabbit early pregnancy factors Journal of Reproduction and Fertility 87 47-53 